\section{Photodynamische Therapie: Nichtonkologische Indikationen}

Photodynamic Therapy: Non-Oncologic Indications

\section{Zusammenfassung}

Während die Wirksamkeit der Photodynamischen Therapie (PDT) in der Behandlung von oberflächlichen, nichtpigmentierten Hauttumoren inzwischen durch klinisch kontrollierte Studien belegt ist, liegen kaum kontrollierte Studien vor, die die Wirksamkeit der PDT in der Behandlung nichtonkologischer Hauterkrankungen zeigen. Zahlreiche viel versprechende Berichte über die PDT von entzündlichen Dermatosen, wie der Psoriasis vulgaris, der zirkumskripten Sklerodermie, dem Lichen sclerosus et atrophicans oder virusinduzierten Hauterkrankungen weisen darauf hin, dass sich die PDT gerade auch für die Behandlung von nichtonkologischen Dermatosen durchsetzen wird - nicht zuletzt da sie zahlreiche Vorteile gegenüber herkömmlichen Therapieoptionen aufweist und mit wenig Nebenwirkungen behaftet ist. Während die PDT von Tumoren auf eine Zerstörung von erkranktem Gewebe zielt, ist die PDT von entzündlichen Erkrankungen auf eine Modulation zellulärer Funktionen gerichtet. Die therapeutischen Protokolle unterscheiden sich daher erheblich von denen zur Tumorzerstörung. Zur Behandlung von entzündlichen Hauterkrankungen werden in der Regel sehr viel niedrigere Photosensibilisator- und Lichtdosen benötigt („lowdose PDT“), dafür sind jedoch meist mehrere Behandlungssitzungen erforderlich, um die gewünschten Effekte zu erzielen. Die meisten Erfahrungen liegen dabei mit dem topisch zu applizierenden, endogenen Photosensensibilisator 5-Aminolävulinsäure vor. Lediglich zur Behandlung der Psoriasis vulgaris wurden auch systemische Photosensibilisatoren angewandt. Kontrollierte klinische Studien sollten die Wirksamkeit der PDT in diesem viel versprechenden Feld entzündlicher Erkrankungen noch weiter untersuchen, geeignete Behandlungsprotokolle etablieren und möglicherweise auch noch weitere, neue Indikationen für die PDT in der Dermatologie erschließen.

\section{Abstract}

While efficacy of photodynamic therapy (PDT) in the treatment of superficial, non-melanoma skin cancer has been proven in controlled clinical trials, there are only few controlled studies showing the usefulness of topical PDT for non-oncologic diseases of the skin. Several promising reports on PDT of inflammatory dermatoses, e.g. psoriasis vulgaris, localized scleroderma, lichen sclerosus et atrophicus or virus induced skin disorders offer promise that PDT will set up in this area also since it offers several advantages over established treatment modalities and is associated with only few side effects. In contrast to PDT of tumors, where the aim of therapy is tumor destruction, PDT of inflammatory disorders probably works by modulating cellular functions. Therapeutic protocols therefore differ markedly from those used for tumor destruction. For treatment of inflammatory dermatoses usually very low photosensitizer and light doses are sufficient ("low-dose PDT"), however multiple treatments are commonly necessary to achieve the desired effects. Most experiences are available with the endogenous topical photosensitizer 5-aminolevulinic acid. Only for the treatment of psoriasis also systemic photosensitizers have been used successfully. Controlled clinical trials are necessary to study the efficacy of PDT in this promising new field of inflammatory disorders, to establish treatment protocols and probably find new indications for PDT in dermatology. 
Einleitung

Die Photodynamische Therapie (PDT) ist ein inzwischen etabliertes Therapieverfahren zur Behandlung von oberflächlichen nichtpigmentierten Tumoren und Präkanzerosen der Haut. Die Anreicherung von Photosensibilisatoren im erkrankten Gewebe ist jedoch nicht spezifisch für maligne oder prämaligne Tumoren, die Aufnahme von Photosensibilisatoren, insbesondere von 5-Aminolävulinsäure, erfolgt auch in benignen proliferativen Veränderungen wie der Psoriasis vulgaris oder viralen Warzen [1]. Zahlreiche viel versprechende Fallberichte zur PDT von entzündlichen Erkrankungen der Haut, wie der Psoriasis vulgaris oder der zirkumskripten Sklerodermie, deuten jedoch darauf hin, dass sich die PDT insbesondere auch in diesem Bereich durchsetzen wird, da sie viele Vorteile gegenüber herkömmlichen Therapieverfahren aufweist und mit wenig Nebenwirkungen behaftet ist.

Im Gegensatz zur PDT von Tumoren, bei der eine Zellzerstörung das Ziel der Therapie ist, spielt bei der PDT von entzündlichen Hauterkrankungen wahrscheinlich die Modulation zellulärer Funktionen eine wesentliche Rolle. Die Therapieprotokolle unterscheiden sich dabei ganz wesentlich von den zur Tumortherapie verwendeten. So sind zur Behandlung von entzündlichen Hauterkrankungen meistens deutlich niedrigere Licht- und Photosensibilisator-Dosen im Sinne einer „low-dose-PDT“ erforderlich, dafür sind aber in der Regel wiederholte Behandlungen notwendig, um den erwünschten Therapieeffekt bei fehlenden oder nur geringfügigen Nebenwirkungen zu erzielen.

Im Folgenden werden die nichtonkologischen Indikationen angeführt, für die Hinweise aus der Literatur auf eine Wirksamkeit der PDT vorliegen (Tab. 1). Die meisten Erfahrungen liegen dabei für die topische PDT mit dem endogenen Photosensibilisator 5-Aminolävulinsäure (ALA) vor. Lediglich bei der Psoriasis vulgaris kommt auch die systemische PDT zum Einsatz.

Tab. 1 Nichtonkologische Indikationen für die Photodynamische Therapie

\begin{tabular}{|lll|}
\hline Kontrollierte Studien & Fall-Serien & Einzelfallberichte \\
\hline Vulgäre Warzen & $\begin{array}{l}\text { Zirkumskripte Sklero- } \\
\text { dermie }\end{array}$ & Kutane Sarkoidose \\
\hline Psoriasis vulgaris & $\begin{array}{l}\text { Lichen sclerosus et } \\
\text { atrophicans }\end{array}$ & Nävus sebaceus \\
\hline Akne vulgaris & $\begin{array}{l}\text { Alopecia areata } \\
\text { Hypertrichose } \\
\text { Kutane Leishmaniose } \\
\text { Condylomata acumi- } \\
\text { nata }\end{array}$ & $\begin{array}{l}\text { Epidermodysplasia ver- } \\
\text { ruciformis }\end{array}$ \\
\hline
\end{tabular}

Indikationen

\section{Psoriasis vulgaris}

Die UV-Lichttherapie ist die Standardtherapie der mittelschweren bis schweren Psoriasis vulgaris, sei es als Kombination der photosensibilisierenden Substanz 8-Methoxypsoralen mit UVA-
Licht (PUVA-Therapie) oder als UVB-Lichttherapie. Der Nachteil dieser Therapie ist jedoch das bekannte karzinogene Potenzial des UV-Lichtes. Mit dem Ziel dieses Risiko zu umgehen, wurde die PDT zur Behandlung der Psoriasis eingesetzt. Dabei wurden sowohl systemische als auch topische Photosensibilisatoren verwendet.

\section{Systemische PDT der Psoriasis}

Erstmals im Jahr 1937 setzte Silver Hämatoporphyrin und UVLicht zur Behandlung der Psoriasis ein [2]. Er injizierte 6 Patienten mit Psoriasis Hämatoporphyrin an mehreren aufeinander folgenden Tagen i.m. und bestrahlte sie anschließend mit UVLicht. Nach 2 Wochen, nach einem erneuten Therapiezyklus und zusätzlicher oraler Gabe des Sensibilisators bildeten sich die Psoriasisplaques deutlich zurück [2].

Im Jahr 1989 publizierte Emtestam die Ergebnisse einer Studie mit 10 Psoriasispatienten, die einer systemischen PDT mit Zinnprotoporphyrin (einmalige Gabe von $2 \mu \mathrm{mol} / \mathrm{kg} \mathrm{KG}$ i.v.) und anschließender Bestrahlung mit UVA-Licht (kumulative UVA-Dosis $98,3 \mathrm{~J} / \mathrm{cm}^{2}$, fraktioniert über 21 Tage) unterzogen wurden [3]. Bei allen Patienten besserten sich die behandelten Psoriasisherde, die mittleren Psoriasis-Scores fielen von 7,9 auf 3,6. Der erfolgreiche Einsatz von UV-Licht durch Emtestam bei der PDT der Psoriasis zeigt, dass rotes Licht mit größerer Eindringtiefe in das Gewebe zur Therapie der Psoriasis nicht unbedingt erforderlich ist. Allerdings wird durch den Einsatz von UV-Licht auf den wesentlichen Vorteil der PDT, nämlich die fehlende Kanzerogenität von rotem Licht, verzichtet. Außerdem stehen die Ergebnisse einer Untersuchung von Weinstein und Mitarbeitern im Widerspruch zur Studie von Emtestam. Weinstein behandelte 8 Patienten mit Psoriasis nach einmaliger i.v. Gabe von $0,5 \mathrm{mg} / \mathrm{kg} \mathrm{KG}$ Photofrin gefolgt von 9-20 Bestrahlungen mit Licht verschiedener Wellenlängen und Lichtdosen [4]. Die Ansprechrate der Psoriasisherde war nach Anwendung von UVA-Strahlern oder Licht von $405 \mathrm{~nm}$ deutlich schlechter als nach Anwendung von Licht mit $630 \mathrm{~nm}$. Das Problem bei der Verwendung von Photofrin ist jedoch die lang andauernde generalisierte, kutane Photosensibilisierung. Aus diesem Grund wurde der systemische Photosensibilisator Benzoporphyrin-derivat-Monosäurering A (Verteporfin) zur Behandlung der Psoriasis eingesetzt, da dieses Porphyrinderivat im Vergleich zu Photofrin eine deutlich kürzere kutanen Photosensibilisierung von 4-7 Tagen zur Folge hat. Levy konnte in einer Phase-I Studie nach i.v. Gabe von $0,2 \mathrm{mg} / \mathrm{kg} \mathrm{KG}$ Verteporfin und Bestrahlung nach 3 Stunden mit rotem Licht $\left(690 \mathrm{~nm}, 75 \mathrm{~J} / \mathrm{cm}^{2}\right.$ ) ein Abheilen der behandelten Psoriasisherde über einen Zeitraum von mindestens 60 Tagen erzielen [5]. Das gute Ansprechen der Psoriasis auf eine systemische PDT mit Verteporfin in Kombination mit Laserlicht der Wellenlänge $690 \mathrm{~nm}$ konnte auch durch Hruza und Mitarbeiter bestätigt werden [6]. Da allerdings für die großflächige Bestrahlung bei ausgedehnter Psoriasis Lasersysteme wenig praktikabel sind, wurde in einer weiteren Studie UVA-Licht für die Bestrahlung verwendet [7]. Verteporfin wurde in einer Konzentration von $8 \mathrm{mg} / \mathrm{m}^{2}$ Körperoberfläche einmal wöchentlich bei 17 Patienten mit mittelschwerer bis schwerer Psoriasis und Psoriasis-Arthritis appliziert, die Bestrahlung erfolgte mit steigenden Dosen von UVALicht (20-80\% der mittleren Erythemdosis nach Verteporfin). Bei 5 von 17 Patienten verbesserte sich der „Psoriasis activity and severity score“ um mindestens 35\%. Die arthritischen Be- 
schwerden besserten sich zwar subjektiv, die objektiven rheumatologischen Parameter veränderten sich jedoch nicht. In einer neueren Studie wurde rotes Licht aus einer inkohärenten Lampe (PDT 1200, Waldmann, Villingen-Schwenningen) für die Bestrahlung verwendet [8]. 15 Patienten mit Psoriasis erhielten fünfmal wöchentlich $8 \mathrm{mg} / \mathrm{m}^{2}$ Körperoberfläche Verteporfin i.v., die Bestrahlung der Psoriasisherde wurde 3 Stunden danach mit $60 \mathrm{~J} / \mathrm{cm}^{2}$ durchgeführt. Die klinischen Psoriasis-Scores sanken von 4,0 auf 2,5 nach 5 PDT-Zyklen. Allerdings trat bei einem Patienten eine Thrombophlebitis infolge der starken Venenreizung bei der Injektion des Photosensibilisators auf.

\section{Topische PDT der Psoriasis}

Durch die topische Anwendung des Photosensibilisators kann eine generalisierte kutane Photosensibilisierung der Haut vermieden werden, so dass dieser Applikationsform zur Behandlung von leicht zugänglichen Hautveränderungen grundsätzlich der Vorzug zu geben ist.

Erstmals im Jahr 1989 beschrieben Meffert und Kollegen die erfolgreiche Behandlung der Psoriasis mittels topischer PDT [9]. In einer placebokontrollierten Pilotstudie an 12 Psoriasis-Patienten wurde Hämatoporphyrinderivat in Form einer Salbe $\left(1 \mathrm{mg} / \mathrm{m}^{2}\right.$ KOF) lokal appliziert. Nach einer Einwirkzeit von einer Stunde erfolgte dann die Bestrahlung mit einer Halogenlampe $\left(\lambda=375-700 \mathrm{~nm}, 3,3 \mathrm{~mW} / \mathrm{cm}^{2}, 2,4,6 \mathrm{~J} / \mathrm{cm}^{2}\right)$, die Behandlung wurde jeweils an 3 aufeinander folgenden Tagen durchgeführt. Eine signifikante Besserung der Psoriasisherde zeigte sich bereits 24 Stunden nach der ersten Behandlung.

Der bisher am besten untersuchte topische Photosensibilisator ist die 5-Aminolävulinsäure (MW 170), die in der Lage ist, das parakeratotische Stratum corneum im Bereich von Psoriasisherden zu durchdringen und sich selektiv in den erkrankten Zellen anzureichern. Die selektive Anreicherung von ALA-induzierten Porphyrinen in psoriatisch veränderter Haut ist in Studien gezeigt worden [10].

Hürlimann und Mitarbeiter erreichten eine partielle Remission von 15 Psoriasisherden nach ein- bis fünfmal durchgeführter topischer PDT mit ALA [11]. Weinstein und Kollegen behandelten die Patienten einmal wöchentlich über 4 Wochen mit $10 \%$ iger, 20\%iger und 30\%iger ALA-Creme und anschließender Bestrahlung mit rotem Licht aus einem Argonionen-gepumpten Farbstofflaser $\left(10-150 \mathrm{~J} / \mathrm{cm}^{2}\right)[12]$. Wiederholte Behandlungen waren dabei deutlich effektiver als eine einmalige Behandlung. Die besten Ergebnisse wurden mit den höheren ALA-Konzentrationen $(20-30 \%)$ erreicht. Boehnke verglich bei 3 Patienten die Wirksamkeit der ALA-PDT mit einer herkömmlichen Dithranolbehandlung im Halbseitenversuch [13]. Die Patienten wurden entweder mit einer 10\%igen ALA-Salbe (Inkubation für 5 Stunden) und rotem Licht aus einer inkohärenten Lichtquelle $\left(600-700 \mathrm{~nm}, 70 \mathrm{~mW} / \mathrm{cm}^{2}, 25 \mathrm{~J} / \mathrm{cm}^{2}\right)$ jeweils dreimal in wöchentlichen Abständen oder auf herkömmliche Weise mit Dithranol behandelt. Die Zeit bis zur Abheilung der Psoriasisherde war in beiden Therapiearmen gleich. In einer neueren Untersuchung von Collins und Mitarbeitern wurden 22 Patienten mit Psoriasis mittels ALA-PDT behandelt [14]. Nach Auftragen einer $20 \%$ igen ALA-Zubereitung für 4 Stunden erfolgte die Bestrahlung mit Licht der Wellenlänge 400-650 nm aus einem Diaprojektor $\left(2-16 \mathrm{~J} / \mathrm{cm}^{2}, 300 \mathrm{~mW} / \mathrm{cm}^{2}\right)$. Bei 7 von 22 Patienten heilten einige der behandelten Herde ab. In einer weiteren Studie untersuchte dieselbe Arbeitsgruppe den Effekt wiederholter Behandlungen mittels ALA-PDT [15]. 10 Patienten mit chronisch-stationärer Psoriasis wurden dabei bis zu dreimal wöchentlich insgesamt bis zu maximal 12-mal behandelt. Eine 20\%ige ALA-Emulsion wurde für 4 Stunden aufgetragen, danach wurden die Herde mit einer Breitband-Lichtquelle mit $15 \mathrm{~mW} / \mathrm{cm}^{2}$ und $8 \mathrm{~J} / \mathrm{cm}^{2}$ bestrahlt. Bei 8 Patienten zeigte sich ein klinischer Erfolg. Allerdings berichteten alle Patienten über Schmerzen während der Behandlung.

Der Stellenwert der PDT in der Behandlung der Psoriasis ist aus der Literatur noch nicht sicher zu bewerten, da sich die bisher verwendeten Therapieprotokolle ganz erheblich unterscheiden und kontrollierte Studien mit entsprechend hohen Fallzahlen fehlen. Zahlreiche Vorteile der PDT gegenüber etablierten Therapieverfahren liegen auf der Hand. Im Vergleich zur UV-Bestrahlung, deren karzinogenes Potential bekannt ist, gibt es bislang keine Hinweise auf ein erhöhtes kutanes Karzinomrisiko nach PDT. Bisher vorliegende Untersuchungen weisen außerdem darauf hin, dass die Anzahl der Behandlungen bis zum Therapieerfolg bei der PDT geringer sind als z.B. bei der PUVA-Therapie. Diesen Vorteilen stehen jedoch auch einige Nachteile gegenüber. Eine in der Literatur beschriebene Nebenwirkung der PDT ist das Auftreten eines so genannten Köbner-Phänomens (isomorpher Reizeffekt) [16]. Ein Patient mit aktinischen Keratosen und spinozellulären Karzinomen wurde mittels topischer ALA-PDT behandelt und entwickelte zwei Tage danach psoriatische Läsionen im Behandlungsareal. Das größte Problem der ALA-PDT sind aber die während der Bestrahlung auftretenden Schmerzen, die abhängig sind von der Licht- und Photosensibilisatordosis und die zum Teil bis zu 2 Tage lang anhalten können. Es bleibt daher zu untersuchen, inwieweit die Photosensibilisator- und Lichtdosen im Sinne des Konzeptes einer „low-dose-PDT“ mit dem Ziel der Schmerzreduktion verringert werden können, ohne die Wirksamkeit der Therapie zu beeinträchtigen.

\section{Infektiöse Hauterkrankungen}

\section{Virusinduzierte Hauterkrankungen}

Durch humane Papillomviren (HPV) verursachte Hauterkrankungen sind besonders häufig und können als vulgäre Warzen an Händen und Füßen, plane Warzen oder Feigwarzen (Condylomata acuminata) in Erscheinung treten. Der klinische Verlauf ist durch eine hohe Rezidivrate gekennzeichnet. Der Bedarf nach neuen, wirksamen Therapieverfahren ist daher gerade bei dieser Erkrankung hoch. Da die schnell proliferierenden Zellen in viralen Akanthomen ALA-induziertes PPIX selektiv im Vergleich zum umgebenden nichtinfizierten Gewebe akkumulieren [17,18], stellt die PDT möglicherweise eine Therapieoption zur Behandlung von Warzen dar. Zudem ist gezeigt worden, dass die ALAPDT antivirale Eigenschaften besitzt [19]. Im Folgenden werden die ersten klinischen, zum Teil kontrollierten Studien zur PDT von Virus-Warzen zusammengefasst.

\section{Vulgäre Warzen}

Amman und Kollegen veröffentlichten 1995 Daten zur topischen ALA-PDT von vulgären Warzen [20]. Allerdings konnte nur bei ei- 
nem von 6 behandelten Patienten eine komplette Abheilung der Warzen innerhalb von 2 Monaten nach einmaliger PDT erzielt werden. Auch Kennedy und Mitarbeiter hatten keinen Erfolg bei der Behandlung von Warzen [21].

Da die unbefriedigende Wirksamkeit möglicherweise auf die unzureichende Penetration von ALA durch die ausgeprägte Hyperkeratose bei kutanen Warzen zurückzuführen ist, versuchten Smetana und Mitarbeiter die Wirksamkeit der ALA-PDT durch Zugabe der Penetrationsverstärker EDTA (2\%) und DMSO (2\%) zu verbessern. Damit waren sie in der Lage, sehr ausgedehnte vulgäre Warzen bei einem nierentransplantierten Patienten zur Abheilung zu bringen. Innerhalb eines Nachbeobachtungszeitraums von 2 Jahren wurde bei diesem Patienten kein Rezidiv beobachtet [19].

Stender und Mitarbeiter führten bei 30 Patienten mit therapieresistenten vulgären Warzen eine erste vergleichende Studie durch. Nach Auftragen einer 20\%igen ALA-Creme für 5 Stunden erfolgte die Bestrahlung mit einem Diaprojektor mit verschiedenen Wellenlängen und einer Lichtdosis von $40 \mathrm{~J} / \mathrm{cm}^{2}$. Vor PDT erfolgte eine Keratolyse der Warzen [22]. Schließlich heilten signifikant mehr Warzen nach einer dreimalig durchgeführten PDT mit weißem Licht (CR 73\%) ab als nach einer dreimaligen PDT mit blauem Licht (CR 28\%), rotem Licht (CR 42\%) oder nach Kryotherapie (CR 20\%). Innerhalb eines Nachbeobachtungszeitraumes von 12 Monaten traten keine Rezidive auf.

Diese Studie wurde gefolgt von einer doppelblinden randomisierten Studie derselben Arbeitsgruppe bei 45 Patienten, die die Ergebnisse der ersten Pilot-Studie bestätigte [23]. Auch hier zeigte sich, dass die ALA-PDT wirksam ist in der Behandlung von therapieresistenten Hand- und Fußwarzen, sofern die Behandlung mit einer entsprechenden Vorbehandlung (Keratolyse) kombiniert wird. Die Bestrahlung wurde mit einer inkohärenten Lichtquelle (Waldmann PDT 1200, 590-700 nm, $50 \mathrm{~mW} / \mathrm{cm}^{2}$, $70 \mathrm{~J} / \mathrm{cm}^{2}$ ) durchgeführt und wurde nach einer und nach 2 Wochen wiederholt. Wenn die Warzen nach 7 Wochen noch bestanden, erfolgte nochmals eine dreimalige Behandlung in wöchentlichen Abständen. Es ergab sich schließlich ein komplettes Abheilen der Warzen in 56\% der mit ALA-PDT behandelten Warzen im Vergleich zu der Placebogruppe (CR 42\%). Überraschenderweise war die Ansprechrate in der Placebogruppe höher als erwartet ( $42 \%$ versus $30 \%$ ). In einer weiteren placebokontrollierten Studie von Fabbrocini und Mitarbeitern wurden 64 plantare Warzen nach Keratolyse mit 20\%igem ALA behandelt, während 57 Warzen nur mit der wirkstofffreien Salbengrundlage behandelt wurden [24]. Die Bestrahlung erfolgte mit einer inkohärenten Lichtquelle $\left(400-700 \mathrm{~nm}, 50 \mathrm{~J} / \mathrm{cm}^{2}\right)$. Die Behandlung wurde bei Bedarf bis zu dreimal innerhalb von 3 Wochen wiederholt. Zwei Monate nach der Behandlung hatten sich 75\% der mittels ALA-PDT behandelten Warzen komplett zurückgebildet, während nur 22,8\% der mit Placebo behandelten Warzen eine komplette Remission zeigten.

Zusammenfassend deuten die Daten kontrollierter Studien darauf hin, dass die topische PDT mit ALA bei therapieresistenten Warzen in Kombination mit einer vorhergehenden Keratolyse eine Alternative zu herkömmlichen Therapieverfahren darstellt. Im Gegensatz zu diversen chirurgischen Verfahren ist nach PDT keine Narbenbildung im Behandlungsareal zu erwarten, was gerade im Bereich der druckbelasteten Fußsohlen als deutlicher Vorteil der PDT anzusehen ist. Als Nachteil der Therapie sind jedoch die zum Teil starken Schmerzen während der Bestrahlung zu nennen, die den breiten Einsatz der PDT, insbesondere bei Kindern, einschränken.

\section{Condylomata acuminata}

Anogenitale Kondylome sind durch eine sehr hohe Rezidivhäufigkeit nach jedweder Therapie gekennzeichnet. Die meisten destruktiven Therapieverfahren wie die elektrokaustische Abtragung oder die Vaporisation mit dem $\mathrm{CO}_{2}$-Laser führen lediglich zur Entfernung des sichtbaren Anteils der Warzen, während subklinische Läsionen nicht entfernt werden. Obwohl bisher nur wenige Untersuchungen zur ALA-PDT von genitalen Warzen vorliegen, ist die PDT bei dieser Indikation ein viel versprechender Ansatz.

In einer Pilotstudie wurde bei 7 Patienten eine 20\%ige ALACreme mit Lidocainhydrochlorid-Zusatz auf anogenitale Warzen aufgetragen. Nach 14 Stunden erfolgte zusätzlich die Applikation einer anästhesierenden Creme für weitere 2 Stunden und anschließend die Bestrahlung mit einem Argonionen-gepumpten Farbstofflaser $\left(630 \mathrm{~nm}, 100 \mathrm{~J} / \mathrm{cm}^{2}, 75-150 \mathrm{~mW} / \mathrm{cm}^{2}\right)$ [25]. Bei 4 der 7 Patienten zeigte sich eine 100\%ige Abheilung der bis dahin therapieresistenten genitalen Warzen.

Da eine selektive Anreicherung von PPIX in den Warzen entscheidend für den Therapieerfolg ist, untersuchten Fehr und Mitarbeiter die Fluoreszenz von PPIX nach topischer Applikation von ALA auf Kondylome im Bereich der Vulva bei 22 Patientinnen [17]. 3 bis 6 Stunden nach Auftragen von ALA zeigte sich eine gleichmäßig verteilte PPIX Fluoreszenz in der Epidermis. Nach 24 Stunden fand sich die Fluoreszenz nur noch im Bereich der Granularzellschicht. Über ähnliche Ergebnisse berichten Ross und Mitarbeiter, die eine selektive Anreicherung von PPIX in Kondylomen nach topischer Applikation von ALA zeigen konnten, wobei die höchste Selektivität gegenüber der normalen Haut bereits 2 Stunden nach Inkubation mit ALA erreicht wurde [18]. Diese Untersuchungen zeigen, dass eine kurze Inkubationszeit von 3 Stunden zu einer uniformen und selektiven Anreicherung von PPIX in den Zielstrukturen führt, während längere Inkubationszeiten von z. B. 14 Stunden, wie in der Studie von Frank et al., eher zu einem Verlust der Selektivität führen.

Das wichtigste Ziel bei der Therapie von anogenitalen Warzen ist eine Verminderung der sehr hohen Rezidivraten nach konventionellen Therapieverfahren. Eine Kombination aus herkömmlichen ablativen Therapieverfahren mit einer PDT, die möglicherweise zusätzlich zu einer selektiven Zerstörung klinisch nichtevidenter, jedoch virusbefallener Areale führt, könnte uns diesem Ziel näher bringen.

\section{Epidermodysplasia verruciformis}

Bei der Epidermodysplasia verruciformis handelt es sich um eine genetisch determinierte Empfänglichkeit für bestimmte Papillomviren. Infolge dessen kommt es bei diesen Patienten zu flachen, warzenähnlichen Hautveränderungen, die maligne entarten können. Eine UV-induzierte Immunsuppression begünstigt die Entstehung epithelialer Hauttumoren, die daher meist in 
lichtexponierten Hautarealen entstehen. Da es keine zufrieden stellende Behandlung für diese seltene Erkrankung gibt, wurde die Wirksamkeit der topischen ALA-PDT untersucht [26]. Bei einer 65-jährigen Patientin bestanden zahlreiche flache Warzen an den Händen, Unterarmen und im Gesicht. Zahlreiche oberflächliche Hauttumoren waren bereits operativ entfernt worden. Die zu behandelnden Läsionen wurden mit einer 20\%igen ALASalbe für 6 Stunden inkubiert und anschließend mit einer inkohärenten Lichtquelle (Waldmann PDT 1200L, Villingen-Schwenningen) mit $160 \mathrm{~J} / \mathrm{cm}^{2}$ und $160 \mathrm{~mW} / \mathrm{cm}^{2}$ bestrahlt. Nach einer einzigen Behandlung heilten die Hautveränderungen komplett ab, das kosmetische Ergebnis war hervorragend. 12 Monate nach der Behandlung waren zwar im Bereich der Hände vereinzelt neue Läsionen aufgetreten, das Gesicht blieb aber weiterhin erscheinungsfrei. Die neuen Herde an den Händen wurden wiederum erfolgreich mittels PDT behandelt. Obwohl durch die PDT keine definitive Heilung dieser genetisch determinierten Erkrankung erreicht werden kann, können die Hautveränderungen doch durch gegebenenfalls in größeren Abständen wiederholt durchgeführte Behandlungen zur Abheilung gebracht werden und damit möglicherweise auch die maligne Entartung im Sinne einer Photochemoprävention verhindert werden.

Die topische Photodynamische Therapie bietet bei der Behandlung von papillomvirusinduzierten Hauterkrankungen verschiedene Vorteile gegenüber herkömmlichen Therapieverfahren. Sie ist nicht invasiv und führt zu hervorragenden kosmetischen Ergebnissen. Die ALA-induzierte Sensibilisierung mit PPXI ist selektiv auf den Ort der Applikation beschränkt, wobei sich virusbefallene, metabolisch veränderte Keratinozyten gegenüber unbefallenen Keratinozyten verstärkt sensibilisieren lassen. Dadurch können zusätzlich makroskopisch noch nicht sichtbare, jedoch virusbefallene Areale eradiziert werden. Ein weiterer Vorteil gegenüber der klassischen $\mathrm{CO}_{2}$-Laservaporisation ist das Fehlen von virusenthaltendem, potentiell infektiösem Laserdampf. Damit können auch Patienten mit infektiösen Erkrankungen wie Hepatitis C oder HIV ohne erhöhtes Risiko für den Operateur behandelt werden.

Die am häufigsten beobachtete Nebenwirkung der ALA-PDT ist auch bei der Behandlung von Feigwarzen der teils starke brennende Schmerz während der Bestrahlung. Ein weiteres Problem stellt die Dosimetrie der Lichtbestrahlung in anatomisch schlecht zugänglichen Regionen dar, wie z.B. bei intraanalen oder vaginalen Läsionen.

\section{Kutane Leishmaniose}

Kürzlich wurde über die erfolgreiche Behandlung von 11 Patienten mit kutaner Leishmaniose durch Leishmania major mittels topischer ALA-PDT berichtet [27]. Nach Applikation einer 10\%igen ALA-Emulsion für 4 Stunden erfolgte die Bestrahlung der Herde mit rotem Licht $\left(570-670 \mathrm{~nm}, 100 \mathrm{~J} / \mathrm{cm}^{2}, 150 \mathrm{~mW} /\right.$ $\mathrm{cm}^{2}$ ). Diese Behandlung wurde in wöchentlichen Abständen solange wiederholt, bis keine Amastigoten im Abstrichpräparat mehr nachweisbar waren. Bereits nach 1-2 Behandlungen waren alle Läsionen bis auf eine frei von Parasiten und zeigten eine deutliche Größenreduktion. Nach Abheilung zeigten die meisten Läsionen lediglich eine oberflächliche Narbenbildung oder leichte postinflammatorische Hyperpigmentierungen. Innerhalb des Nachbeobachtungszeitraumes von 3-6 Monaten traten keine
Rezidive auf. Die einzige Läsion, die nicht zur Abheilung gebracht werden konnte, war ulzeriert und reicherte im nekrotischen Zentrum der Läsion kein ALA bzw. PPIX an. Obwohl diese Läsion deutlich kleiner wurde, konnten im Abstrichpräparat nach zweimaliger PDT noch Amastigoten nachgewiesen werden.

Diese Studie zeigt erstmals die klinische Wirksamkeit der PDT in der Behandlung von parasitären Hauterkrankungen. Diese viel versprechenden Ergebnisse sollten durch kontrolliert randomisierte Studien belegt werden.

\section{Erkrankungen der Talgdrüsen und Haarfollikel}

Die selektive Anreicherung von bestimmten Photosensibilisatoren in Talgdrüsen und Haarfollikeln lässt vermuten, dass die PDT bei der Behandlung von Erkrankungen im Bereich dieser Strukturen, wie z.B. der Hypertrichose, der Alopezie oder der Akne vulgaris, wirksam sein könnte.

\section{Alopecia areata}

So wurde beobachtet, dass am Ort der intramuskulären Injektion von Hämatoporphyrin eine Hypertrichose aufgetreten ist [28]. Monfrecola und Mitarbeiter behandelten daher 2 Patienten mit Alopecia areata mittels topisch appliziertem Hämatoporpyhrin und langwelligem UVA-Licht dreimal pro Woche [29]. Nach einer Therapie über 3-4 Monate kam es zum Wiederwachsen der Haare im Behandlungsareal, die Kontrollareale blieben unverändert. Eine Behandlung der Alopecia areata mittels ALA-PDT (5\%, $10 \%$ und $20 \%$ ALA für 3 Stunden, Bestrahlung mit rotem Licht) blieb hingegen bei 6 Patienten auch nach 20 Behandlungen ohne Erfolg [30].

\section{Hypertrichose}

Die selektive Anreicherung von PPIX im Haarfollikelepithel nach topischer Applikation von ALA stellt theoretisch auch eine ideale Voraussetzung für eine selektive Destruktion von Haarfollikeln dar und somit zu einer bleibenden Entfernung von störenden Haaren, unabhängig von der Haarfarbe oder dem Hauttyp. In einer Pilotstudie wurden 11 Patienten nach vorhergehender Epilation der Haare mit Wachs mittels topischer ALA-PDT behandelt [31]. ALA wurde dabei in einer Konzentration von $10 \%$ oder $20 \%$ für 3 Stunden lokal appliziert und dann mit Laserlicht $(630 \mathrm{~nm})$ einer Lichtdosis von 100 oder $200 \mathrm{~J} / \mathrm{cm}^{2}$ bestrahlt. Zum Zeitpunkt der Nachbeobachtung nach 6 Monaten waren die Kontrollareale und die mit den niedrigeren Dosen behandelten Areale (10\% ALA mit 100 und $200 \mathrm{~J} / \mathrm{cm}^{2}$ bzw. 20\% ALA mit $100 \mathrm{~J} / \mathrm{cm}^{2}$ ) zu mehr als $90 \%$ wieder behaart. Hingegen fand sich in den Arealen, die mit $20 \%$ ALA und $200 \mathrm{~J} / \mathrm{cm}^{2}$ behandelt wurden, eine Reduktion der Haardichte um 40\% nach einer einzigen Behandlung. Während der Bestrahlung berichteten die Patienten allerdings über mäßige bis starke Schmerzen und es kam bei einem Teil der Patienten zu einer vorübergehenden Hyperpigmentierung im Behandlungsareal. Für die mögliche Wirksamkeit der Hochdosis-PDT zur Haarentfernung spricht auch die eigene Beobachtung, dass es bei einzelnen Patienten nach ALA-PDT von Hauttumoren zum bleibenden Haarverlust im Behandlungsareal gekommen ist. Dies muss vor allem auch als potentielle Nebenwirkung der ALA-PDT von Hauttumoren im Bereich des behaarten Kopfes bedacht werden. 
Obwohl die ALA-PDT bei Anwendung hoher Licht- und Photosensibilisator-Dosen zu einem teilweisen bleibenden Verlust von Haaren führen kann, sind die bisher berichteten Ergebnisse sowohl hinsichtlich der Wirksamkeit als auch der beobachteten Nebenwirkungen nicht optimal. So bleibt zu untersuchen, ob diese Therapieansätze durch eine Optimierung der Behandlungsparameter verbessert werden können.

\section{Erkrankungen der Talgdrüsen}

Ähnlich wie in den Haarfollikeln wird ALA auch selektiv in Talgdrüsen aufgenommen und verstoffwechselt [32]. Nach systemischer Gabe von ALA kommt es bei Mäusen zu einem selektiven phototoxischen Schaden von Talgdrüsen und Haarfollikeln. Somit sind Erkrankungen der Talgdrüsen, wie z.B. die Akne vulgaris, Talgdrüsenhyperplasien oder der Nävus sebaceus potentielle Indikationen für eine ALA-PDT.

\section{Nävus sebaceus}

Dierickx und Mitarbeiter behandelten einen Patienten mit einem ausgeprägten Nävus sebaceus im Gesicht mittels ALA-PDT [33]. Die Behandlung (20\% ALA für 4 Stunden, Bestrahlung mit einem Argonionen-gepumpten Farbstofflaser bei $630 \mathrm{~nm}$ mit $100 \mathrm{~J} / \mathrm{cm}^{2}$, $50 \mathrm{~mW} / \mathrm{cm}^{2}$ ) wurde alle 4-8 Wochen insgesamt 13-mal durchgeführt. Nach jeder Behandlung kam es zu einer Regression des Nävus, der jeweils flacher und kleiner wurde, bis er sich schließlich ohne Hinterlassung von Narben weitgehend zurückgebildet hatte. Innerhalb von 16 Monaten kam es zu keinem Rezidiv im Behandlungsareal. Eine nach Ende der Therapie durchgeführte Hautbiopsie zeigte jedoch eine Persistenz der tiefen Anteile des Nävus sebaceus, die möglicherweise zu einem Rezidiv mit der Gefahr der Entstehung der mit diesem Nävus assoziierten Hauttumore führen könnten.

\section{Akne vulgaris}

In einer offenen, prospektiven Studie wurden 22 Patienten mit Akne vulgaris am Rücken ein- bzw. viermal mittels ALA-PDT behandelt [34]. Eine 20\%ige ALA-Zubereitung wurde für 3 Stunden okklusiv aufgetragen, dann erfolgte die Bestrahlung mit rotem Licht ( $550-700 \mathrm{~nm}, 150 \mathrm{~J} / \mathrm{cm}^{2}$ ). Nach der ALA-PDT trat eine selektiv auf die Talgdrüsen begrenzte phototoxische Reaktion auf, die Talgdrüsenfunktion war nachhaltig eingeschränkt und auch die Bakterienbesiedelung in den Follikeln nahm ab. Histologisch zeigte sich ein akuter zytotoxischer Schaden der Talgdrüsen. Klinisch zeigte sich eine signifikante Besserung der entzündlichen Akne nach ALA-PDT, die nach mehrfach durchgeführter PDT über 20 Wochen anhielt und nach einmaliger Therapie über 10 Wochen andauerte. Obwohl die ALA-PDT sich als wirksam zur Behandlung der Akne erwies, traten erhebliche Nebenwirkungen auf. Jede Behandlung war für den Patienten schmerzhaft und führte zu einem akuten Erythem und Ödem, manchmal sogar zur Blasenbildung und Purpura oder zu einem akuten akneiformen Schub. Bei den meisten Patienten trat eine Hyperpigmentierung im Behandlungsareal auf, die erst nach Wochen oder Monaten zurückging. Diese Ergebnisse wurden auch durch eine neuere Untersuchung bestätigt [35]. 23 Patienten mit therapieresistenter Akne vulgaris im Gesicht wurden mittels ALA-PDT (20\%ige ALA-Emulsion für 4 Stunden, Bestrahlung mit einer Halogenlampe mit $17 \mathrm{~mW} / \mathrm{cm}^{2}$ und $13 \mathrm{~J} / \mathrm{cm}^{2}$ ) behandelt. Bei allen Patienten kam es zu einer Besserung der Akne im Gesicht und zu einer Abnahme neuer Akneeffloreszenzen 1, 3 und 6 Monate nach PDT. Als Nebenwirkungen traten aber auch hier Brennen und Stechen während der Bestrahlung, Erytheme und Ödeme für 3 Tage nach PDT sowie eine Exfoliation, Irritation und Überempfindlichkeit gegenüber mechanischen Reizen auf. Nach Abschuppung kam es zu Erythemen oder Hyperpigmentierungen, die bis zu einem Monat andauerten.

Die vorliegenden Untersuchungen zeigen zwar die Wirksamkeit der ALA-PDT in der Behandlung von Erkrankungen der Talgdrüsen und Haarfollikel, die dabei zum Teil auftretenden Nebenwirkungen rechtfertigen derzeit jedoch noch nicht den routinemäßigen Einsatz der PDT für die Behandlung dieser Indikationen.

\section{Sklerosierende Hauterkrankungen Zirkumskripte Sklerodermie}

Bei der zirkumskripten Sklerodermie handelt es sich um eine chronische Erkrankung der Haut, bei der es nach einer entzündlichen Phase zur Sklerose umschriebener Hautareale kommt. Obwohl die PUVA- bzw. Bade-PUVA-Therapie sowie die Hochdosis-UVA ${ }_{1}$-Therapie in der Behandlung der zirkumskripten Sklerodermie wirksam sind, müssen die Langzeitnebenwirkungen der UV-Therapie, insbesondere das karzinogene Potenzial bedacht werden.

In einer klinischen Untersuchung wurden bisher 10 Patienten mit zirkumskripter Sklerodermie, bei denen herkömmliche Therapieverfahren (Bade-PUVA, Penicillin-Infusionen, lokale Therapien) ohne Erfolg geblieben waren, mittels topischer Photodynamischer Therapie mit ALA behandelt. Nach lokaler Applikation einer 3\%igen ALA-Gelzubereitung für 6 Stunden erfolgte die Bestrahlung mit einer inkohärenten Lichtquelle (PDT 1200L, $10 \mathrm{~J} / \mathrm{cm}^{2}, 40 \mathrm{~mW} / \mathrm{cm}^{2}$ ) [36]. Die Behandlung wurde ein- bis zweimal wöchentlich über 3 bis 6 Monate durchgeführt. Die mittlere Anzahl der Behandlungen betrug dabei $26 \pm 8$. Die Sklerodermieherde wurden vor, während und nach Abschluss der Therapie sowie im weiteren Follow-up durch eine quantitative Messung der Hauthärte mittels Durometer [37] sowie durch einen klinischen Hauthärte-Score [38] beurteilt. Bei allen Patienten kam es bis zum Ende der Therapie zu einer signifikanten Reduktion des quantitativen Durometer-Scores sowie zu einer signifikanten Abnahme des qualitativen Hauthärte-Scores (Abb.1 -3). Als Nebenwirkungen der Therapie traten manchmal ein leichtes Brennen oder Juckreiz während der Bestrahlung sowie eine transiente Hyperpigmentierung im Behandlungsareal auf. Bis zu einem Nachbeobachtungszeitraum von etwa 2 Jahren kam es bei den Patienten im Bereich der behandelten Areale zu keiner weiteren Progression der durch die Therapie deutlich gebesserten oder weitgehend abgeheilten sklerotischen Herde. Allerdings konnte das Auftreten neuer Herde bis dato an unbehandelten Lokalisationen nicht verhindert werden.

Die Pathogenese der Sklerodermie beruht auf einer exzessiven Kollagenablagerung in der Dermis der läsionären Haut, während die für den Kollagenabbau verantwortlichen Matrixmetalloproteinasen (MMPs) vermindert sind. In vitro konnte gezeigt werden, dass es durch die PDT mit ALA in humanen dermalen Fibroblasten zu einer Induktion von MMP-1 und MMP-3 kommt, während die Synthese von Kollagen Typ I signifikant reduziert wird [39]. Die Induktion kollagenabbauender Enzyme bei gleichzeitiger Reduktion der Kollagenproduktion ist somit wahrscheinlich 


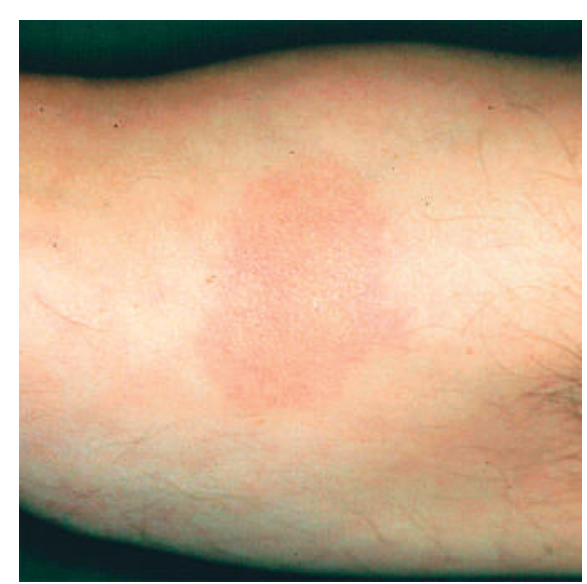

Abb. 1 41-jähriger Mann mit zirkumskripter Sklerodermie am rechten Oberarm.

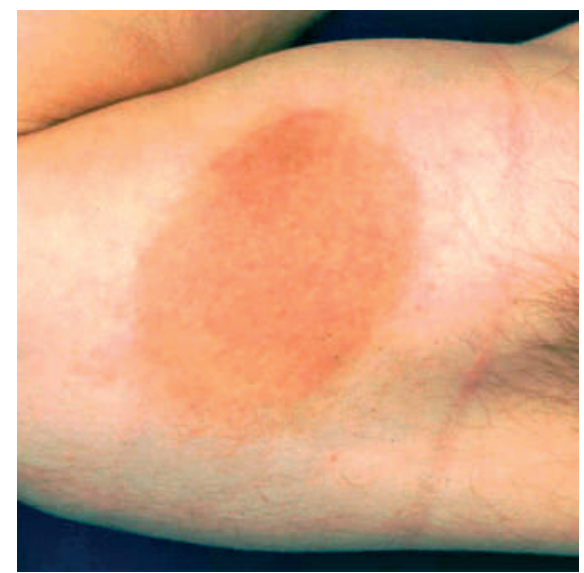

Abb. 2 Gleicher Patient nach 6 Behandlungen mittels ALAPDT und deutlicher Hyperpigmentierung im Behandlungsareal.

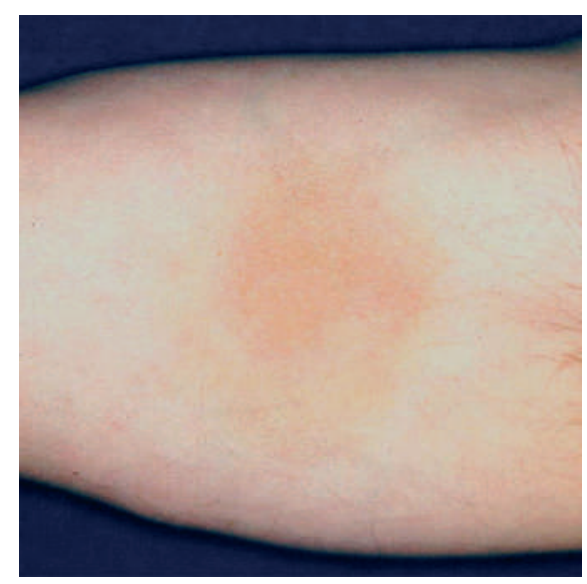

Abb. 3 Gleicher Patient nach insgesamt 25 Behandlungen mittels ALA-PDT. Weitgehende Abheilung des sklerotischen Herdes unter Hinterlassung einer transienten Hyperpigmentierung.

für die beobachteten antisklerotischen Effekte der PDT verantwortlich.

\section{Lichen sclerosus et atrophicans}

Auch eine andere sklerosierende Erkrankung der Haut, der Lichen sclerosus et atrophicans der Vulva, konnte erfolgreich mittels topischer ALA-PDT behandelt werden [40]. 12 Frauen mit stark juckendem Lichen sclerosus wurden mit einer 20\%igen ALA-Zubereitung und Licht aus einem Argonionen-gepumpten Farbstofflaser (Wellenlänge $635 \mathrm{~nm}, 80 \mathrm{~J} / \mathrm{cm}^{2}, 70 \mathrm{~mW} / \mathrm{cm}^{2}$ ) behandelt. Wenn der Pruritus nach der ersten PDT persistierte, wurden die Patientinnen innerhalb von 1-3 Wochen ein zweites Mal behandelt. Die Behandlung wurde gut vertragen, 6-8 Wo- chen nach der letzten PDT hatte sich der Juckreiz bei 10 von 12 Patientinnen weitgehend zurückgebildet.

\section{Kutane Sarkoidose}

Bei der Sarkoidose handelt es sich um eine systemische Erkrankung unbekannter Ätiologie, bei der es zur Bildung von nichtverkäsenden epitheloidzelligen Granulomen in zahlreichen Organen kommen kann. Eine 67-jährige Patientin litt seit über 17 Jahren an einer disseminierten kutanen Sarkoidose ohne weitere systemische Beteiligung. Da verschiedenste Therapieversuche ohne Erfolg geblieben waren und die Patientin sehr unter den rot-braunen kleinknotigen Hautveränderungen an den Beinen litt, wurde ein Therapieversuch mittels topischer ALA-PDT durchgeführt. Nach topischer Applikation einer 3\%igen ALA-Gelzubereitung für 6 Stunden unter Okklusion auf die zu behandelnden Areale erfolgte die Bestrahlung mit einer inkohärenten Lichtquelle (PDT 1200L, $40 \mathrm{~mW} / \mathrm{cm}^{2}, 10-20 \mathrm{~J} / \mathrm{cm}^{2}$ ). Die Behandlung wurde ein- bis zweimal wöchentlich über einen Zeitraum von 3 Monaten durchgeführt. Unter der Behandlung kam es zu einer deutlichen inflammatorischen Reaktion jeweils nach den Bestrahlungen sowie zu einem leichten Brennen während der Bestrahlung. Nach Abschluss der Therapie bildeten sich die Hautveränderungen vollständig unter Hinterlassung einer transienten Hyperpigmentierung zurück. Eine aus dem Behandlungsareal 4 Wochen nach PDT entnommene Hautbiopsie zeigte normale Haut [41].

Dies ist der erste Fallbericht über die erfolgreiche Behandlung einer granulomatösen Hauterkrankung mittels PDT. Auch bei dieser Erkrankung unbekannter Ätiologie spielt wahrscheinlich eine abnorme Immunregulation eine pathogenetische Rolle, die durch die ALA-PDT offensichtlich positiv beeinflusst werden kann. Die Wirkmechanismen, die zu einer Abheilung der kutanen Sarkoidose führen, sind jedoch bisher nicht näher untersucht worden.

\section{Ausblick}

Bisher weisen nur wenige, meist unkontrollierte klinische Studien oder Einzelfallberichte auf die Wirksamkeit der PDT in der Behandlung von nichtonkologischen Erkrankungen der Haut hin. Im Gegensatz zur Therapie von Tumoren ist bei der Behandlung von entzündlichen Hauterkrankungen das Ziel der Therapie in der Regel nicht eine Zerstörung des Gewebes, sondern eher die Modulation zellulärer Funktionen. Daher unterscheiden sich auch die Therapieparameter (Photosensibilisator- und Lichtdosis) und Therapieprotokolle (Anzahl und Dauer der Behandlungen) ganz entscheidend von denen der Tumortherapie. Die genauen Behandlungsparameter für die einzelnen Indikationen sind jedoch bislang nicht durch kontrollierte Studien evaluiert und unterscheiden sich in der Literatur teils ganz erheblich. Manche der nichtonkologischen Hauterkrankungen, wie die Psoriasis, die zirkumskripte Sklerodermie, granulomatöse Erkrankungen oder die Akne vulgaris scheinen auf wiederholt durchgeführte Therapien mit teils sehr niedrigen Licht- und Photosensibilisator-Dosen anzusprechen (low-dose-PDT). Dagegen erscheint bei der Therapie von HPV-induzierten Warzen oder bei der Behandlung der Hypertrichose eine Hochdosis-PDT, analog der Tumorbehandlung, sinnvoller. 
Der Stellenwert der PDT in der Behandlung von nichtonkologischen Indikationen wird erst definiert werden können, wenn entsprechende kontrolliert-randomisierte Studien vorliegen, wie sie beispielhaft bereits für vulgäre Warzen durchgeführt wurden $[23,24]$.

\section{Literatur}

${ }^{1}$ Fritsch C, Lehmann P, Stahl W, Schulte KW, Blohm E, Lang K, Sies H, Ruzicka T. Optimum porphyrin accumulation in epithelial skin tumours and psoriatic lesions after topical application of delta-aminolaevulinic acid. Br J Cancer 1999; 79: $1603-1608$

2 Silver H. Psoriasis vulgaris treated with hematoporpyhrin. Arch Dermatol Syph 1937; 36: 1118 -1119

${ }^{3}$ Emtestam L, Berglund L, Angelin B. Tin-protoporphyrin and long wavelength ultraviolet light treatment of psoriasis. Lancet 1989; 1: $1231-1233$

${ }^{4}$ Weinstein GD, McCullough JL, Nelson JS, Berns MW, McCormick AJ. Low-dose photofrin II photodynamic therapy of psoriasis. Clin Res 1991; 39: 509

${ }^{5}$ Levy JG, Jones CA, Pilson LA. The preclinical and clinical development and potential application of benzoporphyrin derivative. International Photodynamics 1994; $1: 3-5$

${ }^{6}$ Hruza L, Lui H, Hruza G, McLean D, Anderson R. Response of psoriasis to photodynamic therapy using benzoporphyrin derivative monacid ring A. Laser Surg Med Suppl 1995; 4: 43

${ }^{7}$ Bissonnette R, McLean DI, Reid G, Kelsall J, Lui H. Photodynamic therapy of psoriasis and psoriatic arthritis with BPD verteporfin. In: Proceedings of the 17th Biennial Congress, International Photodynamic Association (p. 73). Nantes, France: 1998

8 Boehncke W-H, Elshorst-Schmidt T, Kaufmann R. Systemic photodynamic therapy improves psoriasis. Arch Dermatol 2000; 136: 271 272

${ }^{9}$ Meffert H, Pres H, Diezel W, Sönnichsen N. Antipsoriatische und phototoxische Wirksamkeit von Hämatoporphyrin-Derivat nach topischer Applikation und Bestrahlung mit sichtbarem Licht. Dermatol Monatsschr 1989; 175: 28-34

${ }^{10}$ Stringer MR, Collins P, Robinson DJ, Stables GI, Sheehan-Dare RA. The accumulation of protoporphyrin IX in plaque psoriasis after topical application of 5-aminolevulinic acid indicates a potential for superficial photodynamic therapy. J Invest Dermatol 1996; 107: 76 - 81

${ }^{11}$ Hürlimann AF, Panizzon RA, Burg G. Topical photodynamic treatment of skin tumors and dermatoses. Dermatology 1994; 3: 327

12 Weinstein GD, McCullough JL, Jeffes EW, Nelson JS, Fong NL, McCormick AJ. Photodynamich therapy of psoriasis with topical delta aminolevulinic acid: A pilot dose ranging study. Photodermatol Photoimmunol Photomed 1994; 10: 92

${ }^{13}$ Boehncke W-H, Sterry W, Kaufmann R. Treatment of psoriasis by topical photodynamic therapy with polychromatic light. Lancet 1994; 343: 801

${ }^{14}$ Collins P, Robinson DJ, Stringer MR, Stables GI, Sheehan-Dare RA. The variable response of plaque psoriasis after a single treatment with topical 5-aminolevulinic acid photodynamic therapy. Br J Dermatol 1997; 137: 743 - 749

15 Robinson DJ, Collins P, Stringer MR, Vernon DI, Stables GI, Brown SB, Sheehan-Dare RA. Improved response to plaque psoriasis after multiple treatments with topical 5-aminolevulinic acid photodynamic therapy. Acta Dermato Venereol 1999; 79: 451 -455

${ }^{16}$ Stender IM, Wulf HC. Köbner reaction induced by photodynamic therapy using delta-aminolevulinic acid. Acta Dermato Venereol 1996; 76: $392-393$

${ }^{17}$ Fehr MK, Chapman CF, Krasieva T, Tromberg BJ, McCullough JL, Berns MW, Tadir Y. Selective photosensitizer distribution in vulvar condyloma acuminatum aftertopical application of 5-aminolevulinic acid. Am J Obstet Gynecol. 1996; 174: 51 - 57

18 Ross EV, Romero R, Kollias N, Crum C, Anderson RR. Selectivity of protoporphyrin IX fluorescence for condylomata after topical application of 5-aminolaevulinic acid: implications for photodynamic treatment. Br J Dermatol 1997; 137: 736 - 742
${ }^{19}$ Smetana Z, Malik Z, Orenstein A, Mendelson E, Ben-Hur E. Treatment of viral infections with 5-aminolevulinic acid and light. Lasers Surg Med 1997; 21: 351 - 358

${ }^{20}$ Ammann R, Hunziker T, Braathen LR. Topical photodynamic therapy in verrucae. A pilot study. Dermatology 1995; 191: 346-347

${ }^{21}$ Kennedy JC, Pottier RH, Pross DC. Photodynamic therapy with endogenous protoporphyrin IX: basic principles and present clinical experience. J Photochem Photobiol B 1990; 6: 143-148

${ }^{22}$ Stender IM, Lock-Andersen J, Wulf HC. Recalcitrant hand and foot warts successfully treated with photodynamic therapy with topical 5-aminolaevulinic acid: a pilot study. Clin Exp Dermatol 1999; 24: $154-159$

${ }^{23}$ Stender IM, Na R, Fogh H, Gluud C, Wulf HC. Photodynamic therapy with 5-aminolaevulinic acid or placebo for recalcitrant foot and hand warts: randomised double-blind trial. Lancet 2000; 355: 963-966

${ }^{24}$ Fabbrocini G, Di Constanzo MP, Riccardo AM, Quarto M, Colasanti A, Roberti G, Monfrecola G. Photodynamic therapy with topical $\delta$-aminolaevulinic acid for the treatment of plantar warts. J Photochem Photobiol B: Biol 2001; 61: $30-34$

${ }^{25}$ Frank RG, Bos JD. Photodynamic therapy for condylomata acuminata with local application of 5-aminolevulinic acid. Genitourin Med. 1996; 72: 70-71

${ }^{26}$ Karrer S, Szeimies RM, Abels C, Wlotzke U, Stolz W, Landthaler M. Epidermodysplasia verruciformis treated using topical 5-aminolaevulinic acid photodynamic therapy. Br J Dermatol 1999; 140: 935-938

${ }^{27}$ Enk CD, Fritsch C, Jonas F, Nasereddin A, Ingber A, Jaffe CL, Ruzicka T. Treatment of cutaneous leishmaniasis with photodynamic therapy. Arch Dermatol 2003; 139: 432 - 434

${ }^{28}$ Leroy D, Deschamps P. Long term side effects after intramuscular injection of hematoporphyrin derivative. Photodermatology 1986; 3 : 197-199

${ }^{29}$ Monfrecola G, D'Anna F, Delfino M. Topical hematoporphyrin plus UVA for treatment of alopecia areata. Photodermatology 1987; 4: 305-306

${ }^{30}$ Bissonnette R, Shapiro J, Zeng H, McLean DI, Lui H. Topical photodynamic therapy with 5-aminolaevulinic acid does not induce hair regrowth in patients with extensive alopecia areata.. Br J Dermatol 2000; 143: 1032 - 1035

${ }^{31}$ Grossman M, Wimberly J, Dwyer P. Photodynamic therapy for hirsutism. Lasers Surg Med Suppl 1995; 7: 44

32 Divaris DXG, Kennedy JC, Poittier RH. Phototoxic damage to sebaceous glands and hair follicles of mice after systemic administration of 5-aminolevulinic acid correlates with localized protoporphyrin IX fluorescence. Am J Pathol 1990; 136: 891 - 897

${ }^{33}$ Dierickx CC, Goldenhersch M, Dwyer P, Stratigos A, Mihn M, Anderson R. Photodynamic therapy for nevus sebaceus with topical $\delta$-aminolevulinic acid. Arch Dermatol 1999; 135: 637-640

${ }^{34}$ Hongcharu W, Taylor CR, Chang Y, Aghassi D, Suthamjariya K, Anderson RR. Topical ALA-photodynamic therapy for the treatment of acne vulgaris. J Invest Dermatol 2000; 115: 183-192

35 Itoh Y, Ninomiya Y, Tajima S, Ishibashi A. Photodynamic therapy of acne vulgaris with topical d-aminolevulinic acid and incoherent light in Japanese patients. Br J Dermatol 2001; 144: 575- 579

${ }^{36}$ Karrer S, Abels C, Landthaler M, Szeimies RM. Topical photodynamic therapy for localized scleroderma. Acta Dermato Venereol 2000; 80: $26-27$

${ }^{37}$ Seyger MMB, van den Hoogen FHJ, De Boo T, De Jong EMGJ. Reliability of two methods to assess morphea: skin scoring and the use of a durometer. J Am Acad Dermatol 1997; 37: 793 - 796

${ }^{38}$ Rook AH, Freundlich B, Jegasothy BV, Perez MI, Barr WG, Jimenez SA et al. Treatment of systemic sclerosis with extracorporal photochemotherapy. Results of a multicenter trial. Arch Dermatol 1992; 128 : $337-346$

${ }^{39}$ Karrer S, Bosserhoff AK, Weiderer P, Landthaler M, Szeimies RM. Influence of 5-aminolevulinic acid and red light on collagen metabolism of human dermal fibroblasts. J Invest Dermatol 2003; 120: 325-331

${ }^{40}$ Hillemanns P, Untch M, Pröve F, Baumgartner R, Hillemanns M, Korell M. Photodynamic therapy of vulvar lichen sclerosus with 5-aminolevulinic acid. Obstet Gynecol 1999; 93: 71 - 74

${ }^{41}$ Karrer S, Abels C, Wimmershoff MB, Landthaler M, Szeimies RM. Successful treatment of cutaneous sarcoidosis using topical photodynamic therapy. Arch Dermatol 2002; 138: 581 - 584 